\title{
Tailoring Aqueous Solubility of Functionalized Single-Wall Carbon Nanotubes over a Wide pH Range Through Substituent Chain Length
}

\author{
Liling Zeng, Lei Zhang, and Andrew R. Barron* \\ Department of Chemistry and Center for Nanoscale Science and Technology, Rice University, \\ Houston, Texas 77005, USA
}

Supplementary Materials 


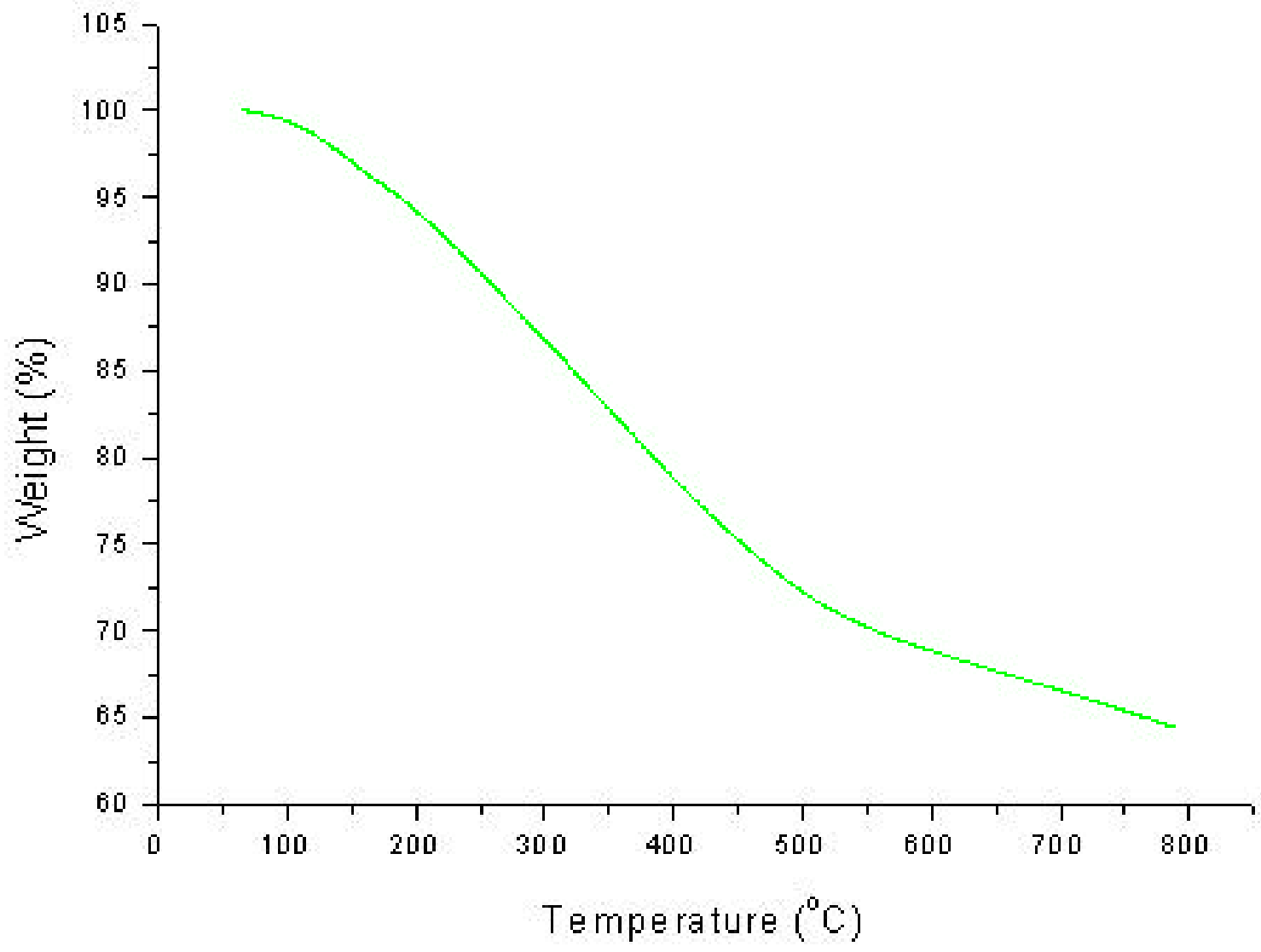

Figure S1. TGA of GLY-SWNT (1). 


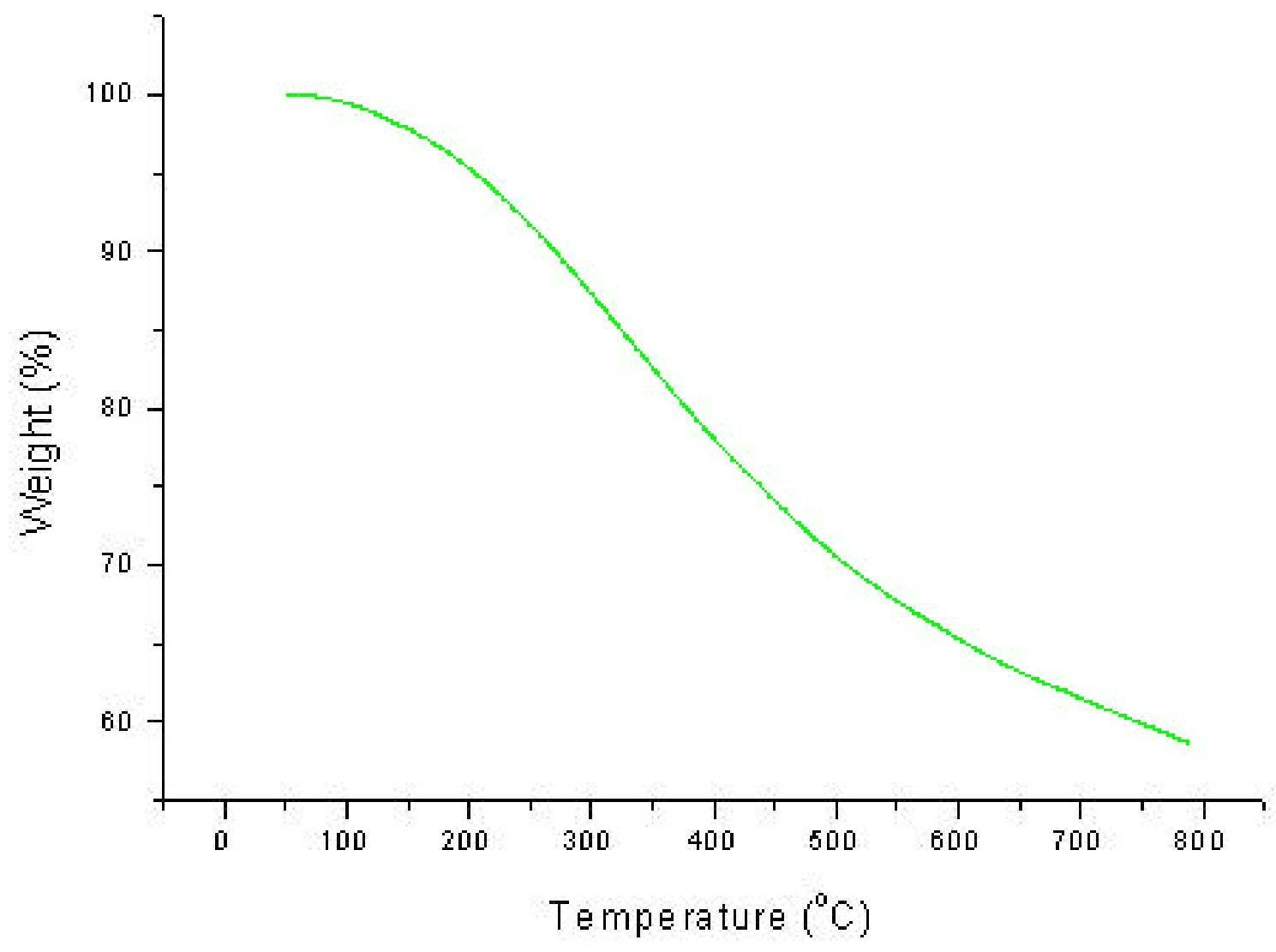

Figure S2. TGA of AHA-SWNT (2). 


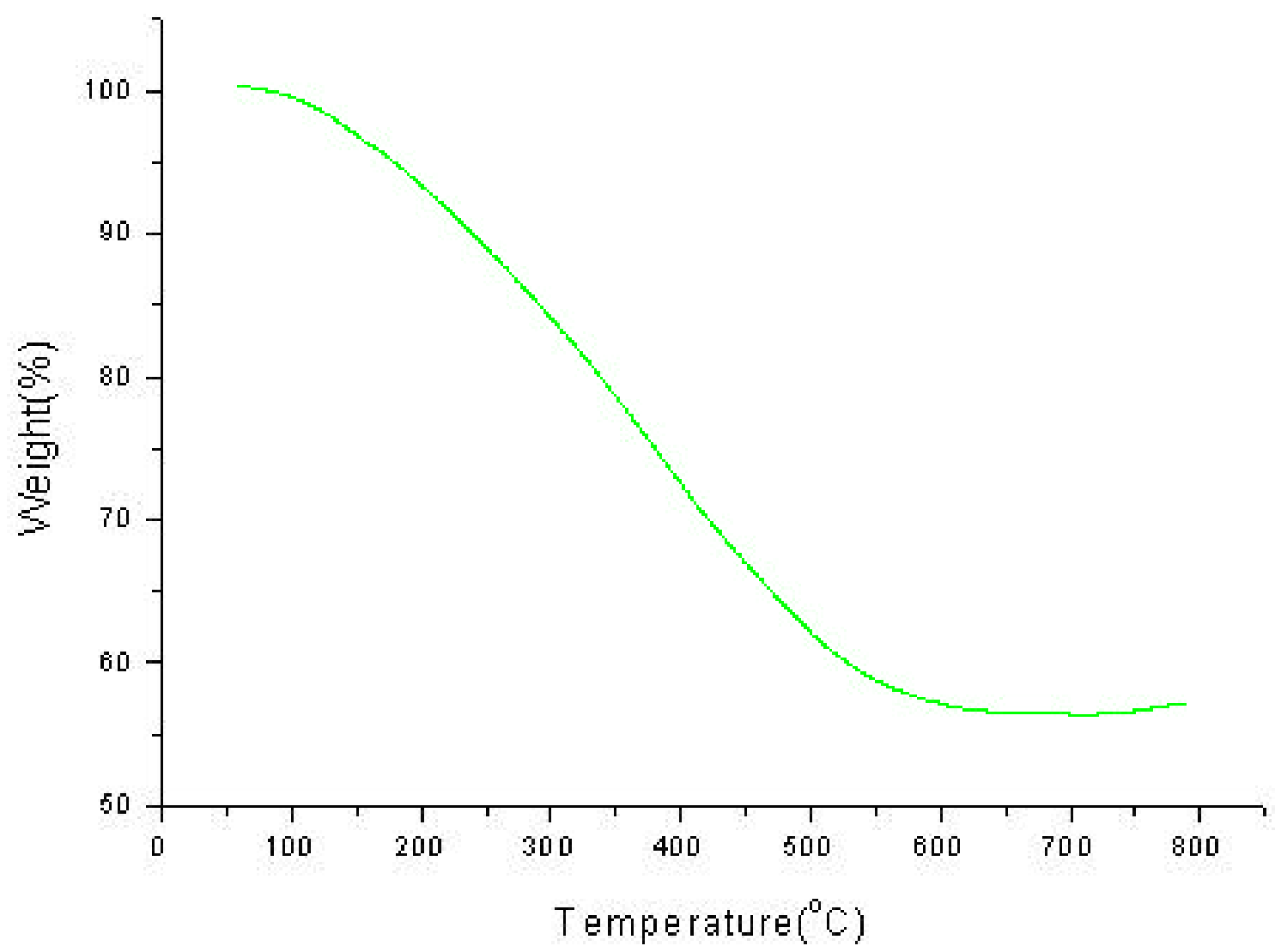

Figure S3. TGA of AUDA-SWNT (3). 


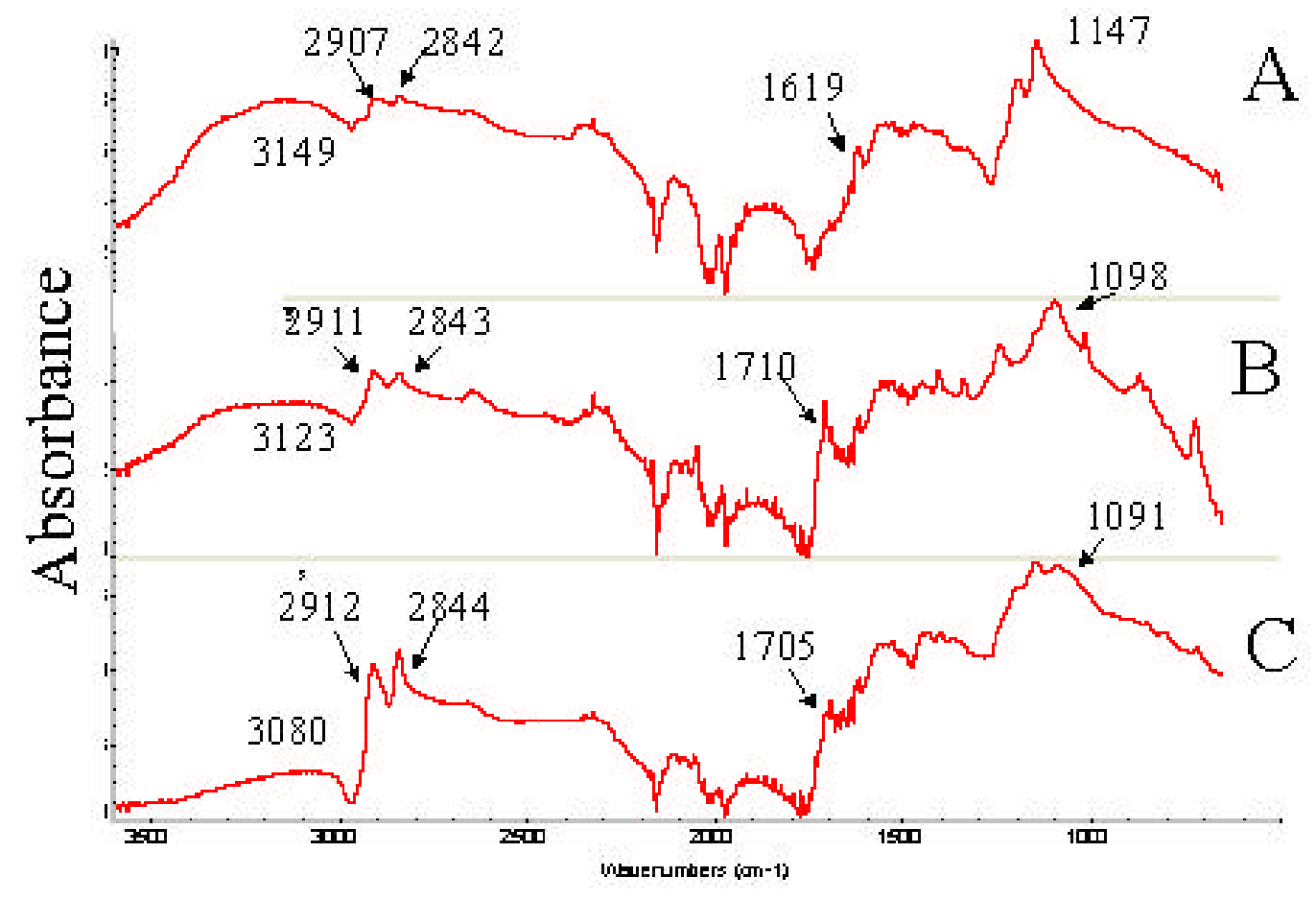

Figure S4. DRIFT-IR spectra of GLY-SWNT (A), AHA-SWNT (B), and AUDA-SWNT (C). 


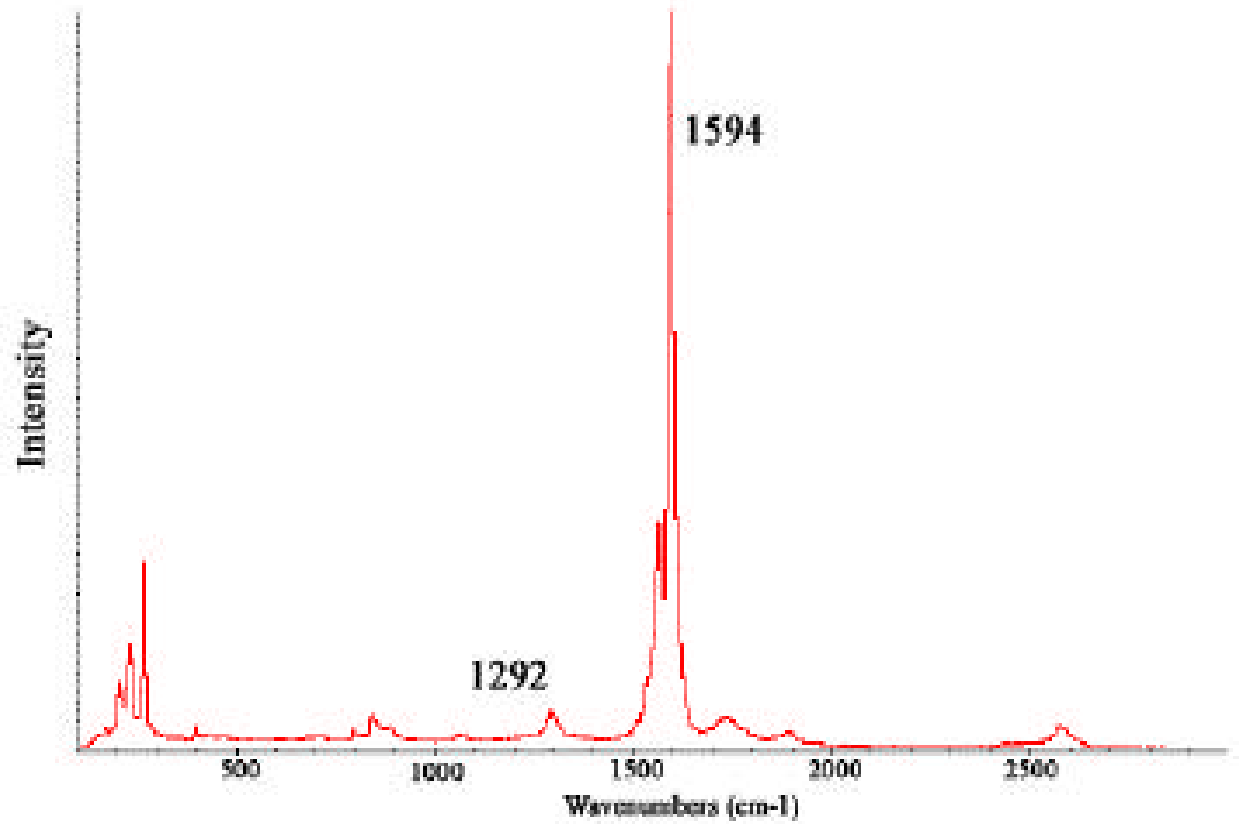

Figure S5. Raman of pristine (unfunctionalized) SWNTs. 